\title{
Mitochondrial DNA copy number variation, leukocyte telomere length, and breast cancer risk in the European Prospective Investigation into Cancer and Nutrition (EPIC) study
}

Daniele Campa', Myrto Barrdahl², Aurelia Santoro33, Gianluca Severi ${ }^{4,5}$, Laura Baglietto ${ }^{4,5,6}$, Hanane Omichessan ${ }^{4,5}$, Rosario Tumino ${ }^{7}$, H. B(as). Bueno-de-Mesquita ${ }^{8,9,10}$, Petra H. Peeters ${ }^{11,12}$, Elisabete Weiderpass ${ }^{13,14,15,16}$, Maria-Dolores Chirlaque 17,18,19, Miguel Rodríguez-Barranco ${ }^{18,19,20}$, Antonio Agudo ${ }^{21}$, Marc Gunter ${ }^{22}$, Laure Dossus ${ }^{22}$, Vittorio Krogh ${ }^{23}$, Giuseppe Matullo ${ }^{24}$, Antonia Trichopoulou ${ }^{25}$, Ruth C. Travis ${ }^{26}$, Federico Canzian ${ }^{27}$ and Rudolf Kaaks ${ }^{2 *}$

\begin{abstract}
Background: Leukocyte telomere length (LTL) and mitochondrial genome (mtDNA) copy number and deletions have been proposed as risk markers for various cancer types, including breast cancer (BC).

Methods: To gain a more comprehensive picture on how these markers can modulate $\mathrm{BC}$ risk, alone or in conjunction, we performed simultaneous measurements of LTL and mtDNA copy number in up to 570 BC cases and 538 controls from the European Prospective Investigation into Cancer and Nutrition (EPIC) cohort. As a first step, we measured LTL and mtDNA copy number in 96 individuals for which a blood sample had been collected twice with an interval of 15 years.

Results: According to the intraclass correlation (ICC), we found very good stability over the time period for both measurements, with ICCs of 0.63 for LTL and 0.60 for mtDNA copy number. In the analysis of the entire study sample, we observed that longer LTL was strongly associated with increased risk of BC $(O R 2.71,95 \% \mathrm{Cl} 1.58-4.65, p=3.07 \times$ $10^{-4}$ for highest vs. lowest quartile; OR 3.20,95\% Cl 1.57-6.55, $p=1.41 \times 10^{-3}$ as a continuous variable). We did not find any association between mtDNA copy number and BC risk; however, when considering only the functional copies, we observed an increased risk of developing estrogen receptor-positive BC (OR 2.47, 95\% Cl 1.05-5.80, $p=0.04$ for highest vs. lowest quartile).
\end{abstract}

Conclusions: We observed a very good correlation between the markers over a period of 15 years. We confirm a role of $L T L$ in $B C$ carcinogenesis and suggest an effect of mtDNA copy number on BC risk.

Keywords: Mitochondrial copy number, Telomere length, Breast cancer, Cancer epidemiology

\footnotetext{
* Correspondence: r.kaaks@dkfz.de

${ }^{2}$ Division of Cancer Epidemiology, German Cancer Research Center/

Deutsches Krebsforschungszentrum (DKFZ), Im Neuenheimer Feld 280, 69120

Heidelberg, Germany

Full list of author information is available at the end of the article
} 


\section{Background}

Mitochondria are responsible for essential functions in the eukaryotic cell, such as energy metabolism, calcium homeostasis, and apoptosis [1, 2]. Mitochondria are also responsible for the generation of ROS, which is a byproduct of energy metabolism. They possess their own genome (mitochondrial DNA [mtDNA]), and in each eukaryotic cell, there can be hundreds or even thousands of copies of their genomes [3]. mtDNA copy number reflects the number of mitochondria within each cell, and this number is maintained within a constant range in order to sustain the energetic needs of the cell [4]. Although mtDNA copy number differs by cell type, it has been observed that there is a good correlation between the number of copies of mtDNA across different cell types within the same individual [5]. Thus, measuring mtDNA copy number in blood has been proposed as a reliable and noninvasive method for estimating the average number of mitochondria in [5].

Over the last decade or so, several reports have suggested that mtDNA content measured in blood may be a risk factor for various diseases, including metabolic diseases, diabetes, obesity, neurodegenerative diseases, and cancer [5-13]. The majority of these studies suggest that high mtDNA copy numbers are associated with increased disease risk. One possible explanation for the association of mtDNA copy number with chronic disease risk is oxidative stress. The mitochondrial genome is particularly prone to DNA damage and mutations caused by oxidative stress, and mtDNA mutations are associated with increased mtDNA copy number as a possible compensatory mechanism to cope with mitochondrial dysfunction.

The study of mtDNA copy number variation in breast cancer $(\mathrm{BC})$ is of particular interest, given the role of oxidative stress in the disease's etiology [14-17]. Only four studies have investigated the role of mtDNA copy number in $\mathrm{BC}$, and these suggested an association between high copy number and increased risk of developing the disease $[5,9,13,18]$. All of these studies were focused entirely or predominantly on estrogen receptor (ER)-positive BC.

mtDNA deletions are a heterogeneous assortment of mtDNA mutations in terms of length and position and represent the major contributor to mitochondrial dysfunction [19]. Along the mtDNA circle, there are hotspot positions for deletions, and $90 \%$ of these include the loss of the nicotinamide adenine dinucleotide dehydrogenase 4 (ND4) sequence [20]. The most frequent deletion is the "common" deletion of $4977 \mathrm{bp}$, including the ND4 sequence, which causes primary mitochondrial diseases but has also been associated with age-related disease susceptibility, such as cancer, muscle atrophy, and neurodegeneration [21, 22]. Moreover, in a recent study, Nie and colleagues reported that the common deletion in the mitochondrial genome could increase the risk of developing $\mathrm{BC}$ [23].

Besides oxidative stress, mitochondrial dysfunction (measured as decreased mitochondrial mass and energy production) recently has been associated with telomere attrition and shortening, and mtDNA copy number has been associated with leukocyte telomere length (LTL) [24]. A possible mechanistic link between telomere length and mitochondrial function has been proposed through the peroxisome proliferator-activated receptor- $\gamma$ pathway [25]. To date, a number of studies have examined LTL in surrogate tissues in relation to cancer risks. However, the results have been inconsistent, showing positive, inverse, or null associations between telomere length and cancer risk, with the majority reporting that shorter telomere length increases the risk (reviewed in [26-29]). In relation to BC risk, studies have shown conflicting results $[26,29]$; however, none of these studies were focused specifically on ER- BC risk.

To date, LTL and mtDNA copy number and deletion levels have been examined mostly as independent contributors to cancer risk. Recent studies have indicated that LTL and mtDNA copy number are positively correlated in healthy individuals, in pregnant women, and in patients with psychological disorders [24, 30, 31]. Moreover, very recently, it has been demonstrated that LTL and mtDNA copy number are correlated in intestinal gastric cancer [32]. Hence, there is emerging evidence that the markers are linked biologically and that their joint measurement may increase their predictive value for cancer risk. Therefore, to gain a more comprehensive picture of how the markers mentioned above can modulate $\mathrm{BC}$ risk, alone or in conjunction, we measured mtDNA copy number, LTL, and mtDNA deletions in the context of the European Prospective Investigation into Cancer and Nutrition (EPIC) cohort. In addition, given the fact that ER- BC has been greatly understudied for these markers, we collected a fairly large number of them $(n=251)$.

\section{Methods}

\section{Study population: the EPIC cohort}

The EPIC cohort has been described in full detail elsewhere [33]. Briefly, EPIC consists of about 520,000 healthy volunteers, aged 35-69 years, who were recruited between 1992 and 2005 in 10 European countries. All EPIC study subjects provided anthropometric measurements (height, weight, and waist and hip circumferences) and extensive standardized questionnaire information about medical history, diet, physical activity, smoking, and other lifestyle factors. The women also answered questions about menstrual and reproductive history, hysterectomy, ovariectomy, and use of exogenous hormones for contraception or treatment of menopausal 
symptoms. About 260,000 women and 140,000 men provided a blood sample, which was split into aliquots of plasma, serum, buffy coat, and erythrocytes and stored frozen for later laboratory analyses.

Cases of cancer occurring after recruitment into the cohort and blood donation are identified through local and national cancer registries or by a combination of contacts with national health insurance and/or active follow-up through the study subjects or their next of kin. Cancer incidence data are classified according to the International Classification of Diseases, Tenth Revision (ICD-10), system. Incident cases of BC were identified as first occurrence of primary invasive tumors, ICD-10 code C50, occurring among women who had no previous diagnosis of cancer. Since 2001, an expanding series of nested case-control studies have been conducted on hormonal, metabolic, and other blood-based risk factors for BC [34-37], as well as on genetic determinants [14, 38-45]. For these latter studies, the cases and controls were not individually matched; however, care was taken to select the controls randomly from the cohort to avoid selection bias. Because there were insufficient amounts of biological material (extracted buffy coat DNA) for some of the selected controls, we retained slightly uneven numbers of invasive $\mathrm{BC}$ cases. We had measurements on telomere length for 570 controls and 533 cases, measurements on mtDNA copy number on 548 controls and 522 cases, and measurements on mtDNA deletions on 539 controls and 519 cases. This study was approved by the ethical review board of the International Agency for Research on Cancer.

\section{Sample preparation and DNA extraction}

All blood specimens were kept frozen in liquid nitrogen at the International Agency for Research on Cancer. DNA was extracted from blood samples on an Autopure instrument (Qiagen, Hilden, Germany) with Puregene chemistry (Qiagen). All of the samples were extracted using the same method and in batches of 96 to avoid any possible bias due to sample handling. The order of DNA from cases and controls was randomized on PCR plates, and each batch cases and controls was chosen to be from the same study center.

\section{LTL and mtDNA copy number/deletion measurement}

LTL was measured by real-time GPCR as the number of copies of telomeric repeats compared with a single copy gene $(R P L P O)$ used as a quantitative control. For each sample plate, a standard curve was prepared by using a serially diluted reference DNA (range 0.468-30 ng/ $\mu \mathrm{l}$ ). The relative length of telomeres $(\mathrm{T})$ compared with the control gene (S) was calculated as the ratio T/S. All the samples were analyzed in triplicate. mtDNA copy number and deletions were also analyzed by qPCR. The
mtDNA/nuclear DNA content was assessed using specific primers designed for the mitochondrial ND1 gene and the nuclear $\beta$-actin gene. The ratio between these two genes $(N D 1 / \beta$-actin) identifies the relative mtDNA copy number [46]. To quantify mtDNA deletions, primers for the mitochondrial gene $N D 4$, located in the major arch of the mtDNA where deletion frequently occurs, were used in combination with those of the mitochondrial gene $N D 1$, which is rarely deleted. The mtDNA deletion level is reported as the ratio between ND4 and ND1 [47] and thus represented by the quantification of the nondeleted fraction of mtDNA (ND4) vs. the total amount of mtDNA (ND1). As for the LTL measurement, for the analysis of mtDNA, a standard curve was also used in each plate. Four samples were included in all plates for all the assays for quality control.

Longitudinal assessment of LTL and mtDNA copy number To assess the reliability of the measure of mtDNA content and LTL over time, we compared the measurements of these two markers obtained from samples collected 15 years apart from a set of 96 samples of healthy individuals randomly selected from among the EPIC Heidelberg cohort. We calculated intraclass correlations (ICCs) by dividing the between-person variance by the total variance (sum of between- and within-person variances) [48]. ICCs $\geq 0.75$ are considered an indicator of excellent reliability; ICCs between 0.51 and 0.74 , good reliability; ICCs between 0.40 and 0.50 , fair reliability; and ICCs $\leq$ 0.40 , poor reliability. This threshold has been selected according to a similar study done on 100 EPIC subjects in which researchers evaluated the reliability of serum metabolite measurements [49].

\section{Statistical analysis}

Quantitative measurements of LTL and mtDNA copy number were log-transformed to obtain variables with an approximately normal distribution. The relationships of age, smoking, and body mass index (BMI) on the biomarkers (LTL and mtDNA copy number/deletion) were examined by linear regression, adjusting for plate number, center, and case-control status. Correlation between the biomarkers (LTL vs. mtDNA copy number, LTL vs. mtDNA, mtDNA copy number vs. deletion) was examined by calculating Pearson's correlation coefficients and corresponding $p$ values.

To examine the relationships of LTL and mtDNA copy number in relation to $\mathrm{BC}$ risk, we used unconditional logistic regression to obtain ORs and 95\% CIs adjusted for age, center, smoking status, BMI, and plate. We also performed stratified analyses by ER status. LTL and mtDNA copy number were analyzed as continuous variables, and as ordinal variables using quartile cutpoints for the variable distributions among the controls. To investigate the 
aggregated effects of mtDNA copy number together with LTL for each individual, the quartiles of the logtransformed values of the markers were added together, creating a score with values ranging from 2 to 8 . The score was investigated as a continuous variable accounting for age, center, and plate. Finally, we investigated the ratio between mtDNA deletion level and mtDNA copy number in relation to $\mathrm{BC}$ risk. This measure represents the proportion of intact molecules relative to the total number of mtDNA (calculated as ND1/nuclear DNA content) molecules per subject.

Considering the number of statistical tests carried out in the present set of analyses, the threshold for statistical significance had to be corrected to avoid false-positive findings. Although it is not reasonable to assume that the three markers are independent, we preferred a conservative approach and thus considered the $p$ value threshold of $(0.05 / 3)<0.017$.

\section{Results}

In the first step, we measured LTL and mtDNA copy number in 96 individuals belonging to the EPIC Heidelberg cohort, from whom a blood sample was collected at 2 time points approximately 15 years apart. Both measurements showed good stability over this 14- to 15-year period, with ICCs of 0.63 for LTL and 0.60 for mtDNA. Given the good reliability of the estimates, we then measured LTL and mtDNA copy number in 570 invasive BC cases and 538 control subjects recruited in the context of the prospective EPIC study. Four samples were included in all plates and analyzed in all assays for quality control. The highest observed coefficient of variation (CV) among these samples and across assays was 5.52\%, and the average $\mathrm{CV}$ over all samples and all assays was $3.06 \%$.

We also calculated CVs on the seven dilutions of the sample used as standard across genotyping plates and assays. The highest observed CV was $3.46 \%$, and the average CV over all samples and all assays was $2.02 \%$.

Regarding the distribution of LTL and mtDNA measures across different strata of risk covariates (Table 1), age was, as expected, inversely related to LTL $(p=1.66$ $\times 10^{-11}$ ) but was not associated with the mtDNA genome variables. Smoking did not show any association with the three markers measured, whereas BMI was significantly associated with mtDNA copy number, though with a modest effect size (correlation coefficient $0.009, p=0.005)$. Similarly to a previous study [24], we also observed a modest but statistically significant, correlation between telomere length and mtDNA copy number (correlation coefficient $0.16, p=7.3 \times 10^{-4}$ ), whereas there was no correlation between the other two markers (Additional file 1: Table S1).

We observed that longer LTL was strongly associated with increased risk of BC when analyzing LTL as a categorical variable (OR 2.71, 95\% CI 1.58-4.65, $p=3.07 \times$ $10^{-4}$ for highest vs. lowest quartile) and also as a continuous variable (OR 3.20, 95\% CI 1.57-6.55, $p=1$. $\left.41 \times 10^{-3}\right)$. When we stratified the analysis by ER status of cancer, we observed that longer telomeres were associated with increased risk of both ER- BC (OR 2.12, $95 \%$ CI 1.04-4.31, $p=3.84 \times 10^{-2}$ for highest vs. lowest quartile) and ER+ BC (OR 2.55, 95\% CI 1.10-5.86, $p=$ $2.82 \times 10^{-2}$ for highest vs. lowest quartile). All results from the fully adjusted analyses on LTL and BC risk are shown in Table 2. (Minimally adjusted models are shown in Additional file 2: Table S2.)

For mtDNA copy numbers, we did not observe any differential association between cases and controls when considering quartiles. Also, the stratified analysis (ER +/ER-) did not show any statistically significant differences between cases and controls (Table 3). The results of the minimally adjusted models are shown in Additional file 3: Table S3. However, we included in the analysis the ratio between mtDNA deletion level and mtDNA copy (ND4/ND1 divided by ND1/nuclear genome). When comparing this measure across cases and controls, we observed an increase in risk with increased number of copies. This difference was statistically significant only for ER+ BC. When analyzed as a categorical variable, unconditional logistic regression models showed a significant association of $\mathrm{ER}+\mathrm{BC}$ with mtDNA integrity (OR 2.47, 95\% CI 1.05-5.80, for highest vs. lowest quartile) (Table 4). The results of the minimally adjusted models are shown in Additional file 4: Table S4. Finally when analyzing the score (computing the aggregate effect of mtDNA copy number and LTL) as a continuous variable, we observed an increased risk for

Table 1 Association between selected markers and age, smoking, and body mass index

\begin{tabular}{|c|c|c|c|c|c|c|}
\hline \multirow[t]{2}{*}{ Marker } & \multicolumn{2}{|l|}{$\mathrm{Age}^{\mathrm{a}}$} & \multicolumn{2}{|l|}{ Smoking $^{b}$} & \multicolumn{2}{|l|}{$\mathrm{BMI}^{\mathrm{b}}$} \\
\hline & Effect (SE) & $p$ value & Effect (SE) & $p$ value & Effect (SE) & $p$ value \\
\hline Telomere length & $-0.004(0.001)$ & $1.6 \times 10^{-5}$ & $-0.002(0.01)$ & 0.84 & $-0.0009(0.002)$ & 0.90 \\
\hline mtDNA copy number & $0.002(0.002)$ & 0.33 & $0.04(0.02)$ & 0.04 & 0.009 (0.0032) & 0.005 \\
\hline mtDNA deletions & $0.0004(0.0008)$ & 0.65 & $-0.01(0.009)$ & 0.10 & $0.001(0.0014)$ & 0.32 \\
\hline
\end{tabular}

BMI Body mass index, mtDNA Mitochondrial DNA

${ }^{\text {aT }}$ The analyses were adjusted for plate and case-control status

${ }^{\mathrm{b}}$ The analyses were adjusted for age, plate, and case-control status 
Table 2 Associations between leukocyte telomere length and breast cancer risk

\begin{tabular}{|c|c|c|c|c|c|c|}
\hline Stratum & Relative $\mathrm{LTL}^{\mathrm{a}}$ & Controls & Cases & OR & $95 \% \mathrm{Cl}$ & $p$ value \\
\hline \multirow[t]{5}{*}{ Overall } & Quartile 1 (0.36-0.63) & 134 & 81 & Reference & - & - \\
\hline & Quartile 2 (0.63-0.75) & 126 & 102 & 1.60 & $(1.04-2.46)$ & 3.17E-02 \\
\hline & Quartile 3 (0.75-0.93) & 134 & 172 & 2.28 & $(1.46-3.57)$ & 2.89E-04 \\
\hline & Quartile 4 (0.93-1.94) & 139 & 215 & 2.71 & $(1.58-4.65)$ & 3.07E-04 \\
\hline & Continuous variable & 533 & 570 & 3.20 & $(1.57-6.55)$ & $1.41 \mathrm{E}-03$ \\
\hline \multirow[t]{5}{*}{ ER+ } & Quartile 1 (0.36-0.63) & 134 & 41 & Reference & - & - \\
\hline & Quartile $2(0.63-0.75)$ & 126 & 45 & 1.59 & $(0.88-2.89)$ & $1.25 \mathrm{E}-01$ \\
\hline & Quartile $3(0.75-0.93)$ & 134 & 77 & 2.97 & $(1.54-5.72)$ & $1.11 \mathrm{E}-03$ \\
\hline & Quartile 4 (0.93-1.94) & 139 & 86 & 2.55 & $(1.10-5.86)$ & $2.82 \mathrm{E}-02$ \\
\hline & continuous variable & 533 & 249 & 3.33 & $(1.17-9.46)$ & $2.38 \mathrm{E}-02$ \\
\hline \multirow[t]{5}{*}{ ER- } & Quartile 1 (0.36-0.63) & 134 & 28 & Reference & - & - \\
\hline & Quartile 2 (0.63-0.75) & 126 & 40 & 1.25 & $(0.65-2.44)$ & 5.05E-01 \\
\hline & Quartile $3(0.75-0.93)$ & 134 & 79 & 1.67 & $(0.88-3.16)$ & 1.19E-01 \\
\hline & Quartile 4 (0.93-1.94) & 139 & 104 & 2.12 & $(1.04-4.31)$ & $3.84 \mathrm{E}-02$ \\
\hline & Continuous variable & 533 & 251 & 2.33 & $(0.88-6.15)$ & 8.92E-02 \\
\hline
\end{tabular}

ER Estrogen receptor, $L T L$ Leukocyte telomere length

${ }^{a}$ Analyses are adjusted for age, body mass index, smoking, center of origin, and plate

individuals with a high score compared with individuals with a low score (OR 1.20, 95\% CI 1.05-1.37, $p=0.009$ ). This trend was present in all the strata but was significant only in the analysis considering ER+ and ER- together (Table 5).

\section{Discussion}

Given their central role in several essential functions of the eukaryotic cell, mitochondria-and in particular mitochondrial mutations-have been extensively investigated in relation to human cancer. A growing number of somatic mitochondrial mutations have been implicated in several cancer types, including $\mathrm{BC}[50,51]$. In addition, the common germline variability in the mitochondrial genome (deletions, mitochondrial single-nucleotide polymorphisms, and resulting mitochondrial haplogroups) has been associated with risk of various cancers, such as of the lung, pancreas, and breast [50, 52-54]. Several studies have suggested that, to counterbalance mitochondrial dysfunction caused by genetic mutations, the eukaryotic cell

Table 3 Associations between mitochondrial DNA copy number and breast cancer risk

\begin{tabular}{|c|c|c|c|c|c|c|}
\hline Stratum & mtDNA copy number ${ }^{a}$ & Controls & Cases & OR & $95 \% \mathrm{Cl}$ & $p$ value \\
\hline \multirow[t]{5}{*}{ Overall } & Quartile $1(0.11-0.21)$ & 132 & 117 & Reference & - & - \\
\hline & Quartile $2(0.21-0.29)$ & 132 & 148 & 1.48 & $(1.00-2.20)$ & $5.24 \mathrm{E}-02$ \\
\hline & Quartile $3(0.29-0.47)$ & 125 & 122 & 1.30 & $(0.81-2.11)$ & 2.77E-01 \\
\hline & Quartile 4 (0.47-1.52) & 133 & 161 & 0.93 & $(0.53-1.66)$ & $8.18 \mathrm{E}-01$ \\
\hline & Continuous variable & 522 & 548 & 0.97 & $(0.66-1.42)$ & $8.75 \mathrm{E}-01$ \\
\hline \multirow[t]{5}{*}{ ER+ } & Quartile $1(0.11-0.21)$ & 132 & 68 & Reference & - & - \\
\hline & Quartile $2(0.21-0.29)$ & 132 & 94 & 1.43 & $(0.85-2.39)$ & 1.77E-01 \\
\hline & Quartile $3(0.29-0.47)$ & 125 & 65 & 1.23 & $(0.65-2.32)$ & $5.22 \mathrm{E}-01$ \\
\hline & Quartile 4 (0.47-1.52) & 133 & 18 & 0.46 & $(0.18-1.13)$ & 8.95E-02 \\
\hline & Continuous variable & 522 & 245 & 0.67 & $(0.36-1.25)$ & 2.11E-01 \\
\hline \multirow[t]{5}{*}{ ER- } & Quartile $1(0.11-0.21)$ & 132 & 41 & Reference & - & - \\
\hline & Quartile $2(0.21-0.29)$ & 132 & 42 & 1.33 & $(0.74-2.38)$ & 3.43E-01 \\
\hline & Quartile $3(0.29-0.47)$ & 125 & 35 & 0.90 & $(0.43-1.91)$ & 7.91E-01 \\
\hline & Quartile 4 (0.47-1.52) & 133 & 140 & 0.94 & $(0.44-2.02)$ & $8.81 \mathrm{E}-01$ \\
\hline & Continuous variable & 522 & 258 & 1.04 & $(0.64-1.69)$ & 8.79E-01 \\
\hline
\end{tabular}


Table 4 Associations between mitochondrial DNA integrity (ND4/ND1 divided by ND1/nuclear genome) and breast cancer risk

\begin{tabular}{|c|c|c|c|c|c|c|}
\hline Stratum & mtDNA integrity ${ }^{a}$ & Controls & Cases & OR & $95 \% \mathrm{Cl}$ & $p$ value \\
\hline \multirow[t]{5}{*}{ Overall } & Quartile $1(0.34-0.90)$ & 129 & 152 & Reference & & \\
\hline & Quartile $2(0.90-1.00)$ & 126 & 102 & 1.06 & $(0.68-1.66)$ & 0.79 \\
\hline & Quartile 3 (1.00-1.11) & 133 & 142 & 1.56 & $(0.95-2.56)$ & 0.08 \\
\hline & Quartile 4 (1.11-2.59) & 130 & 142 & 1.20 & (0.69-2.08) & 0.52 \\
\hline & Continuous variable & 519 & 539 & 1.07 & $(0.75-1.52)$ & 0.73 \\
\hline \multirow[t]{5}{*}{ ER+ } & Quartile $1(0.34-0.90)$ & 129 & 20 & Reference & - & - \\
\hline & Quartile $2(0.90-1.00)$ & 126 & 50 & 1.61 & $(0.78-3.35)$ & 0.20 \\
\hline & Quartile 3 (1.00-1.11) & 133 & 79 & 2.59 & $(1.19-5.66)$ & 0.02 \\
\hline & Quartile 4 (1.11-2.59) & 130 & 89 & 2.47 & $(1.05-5.80)$ & 0.04 \\
\hline & Continuous variable & 519 & 238 & 1.54 & $(0.87-2.72)$ & 0.14 \\
\hline \multirow[t]{5}{*}{ ER- } & Quartile 1 (0.34-0.90) & 129 & 128 & Reference & - & - \\
\hline & Quartile $2(0.90-1.00)$ & 126 & 40 & 0.98 & $(0.55-1.74)$ & 0.94 \\
\hline & Quartile 3 (1.00-1.11) & 133 & 47 & 1.22 & $(0.62-2.39)$ & 0.56 \\
\hline & Quartile 4 (1.11-2.59) & 130 & 41 & 0.96 & $(0.46-2.00)$ & 0.92 \\
\hline & Continuous variable & 519 & 256 & 0.99 & $(0.63-1.54)$ & 0.95 \\
\hline
\end{tabular}

ER Estrogen receptor, $m t D N A$ Mitochondrial DNA

${ }^{a}$ Analyses are adjusted for age, body mass index, smoking, center of origin, and plate

increases their number. Mitochondrial copy number has been proposed as a risk marker for various cancer types $[5-7,9,10,12,13]$. In a small exploratory study on BC, Shen and colleagues reported an association between high mtDNA copy number and increased risk of the disease [5]. This association was subsequently replicated in two independent studies focused primarily on ER+ BC $[9,13]$ and again by Shen and colleagues [18].

Telomeres are specialized structures that adorn the telomeric end of the eukaryotic chromosomes and are essential for the correct segregation of chromosomes to daughter cells [55] as well as for the control of chromosomal stability and regulation of cell growth [56-58]. In the past several years, there has been an overwhelming number of studies aimed at understanding the role of telomere length, measured in blood, in relation to cancer risk and progression. The results, however, are often inconclusive, with some articles reporting an association between increased risk of developing various cancer types and longer telomeres and others reporting the opposite, no association at all, or even a nonlinear dose-response association with increased risks only at

Table 5 Associations between a biomarker score created from leukocyte telomere length and mitochondrial DNA copy number and breast cancer risk

\begin{tabular}{llllll}
\hline Stratum & Controls & Cases & OR & $95 \% \mathrm{Cl}$ & $p$ value \\
\hline Overall & 522 & 548 & 1.20 & $(1.05-1.37)$ & 0.009 \\
ER+ & 522 & 245 & 1.18 & $(0.96-1.45)$ & 0.11 \\
ER- & 522 & 258 & 1.16 & $(0.98-1.38)$ & 0.09
\end{tabular}

ER Estrogen receptor the two extremes of the LTL distribution (very short and very long LTL) [26-29].

LTL and mtDNA copy number have largely been examined as independent contributors to cancer risk; yet, there is emerging evidence that the markers are linked biologically or at least that measuring them together increases the efficiency of the estimation of their effect. For example, Bao and colleagues [59] analyzed LTL and mtDNA as progression markers for hepatocellular carcinoma and observed that the combination of the two was a better indicator than each of the two separately. Moreover, it has been demonstrated that LTL and mtDNA copy number are correlated in intestinal gastric cancer [32]. Recently, an association between mitochondrial dysfunction and telomere length attrition has been suggested, and a correlation between LTL and mtDNA has been observed by several authors [24, 31, 60]. Both telomere length and mitochondrial function have been proposed as markers of aging (although the evidence is much stronger for telomeres). Mice null for either the telomerase reverse transcriptase (Tert) or telomerase RNA component (Terc) gene had marked repression of peroxisome proliferator-activated receptor- $\gamma$, coactivator 1 alpha and beta, and the downstream network. Consistent with peroxisome proliferator-activated receptors as master regulators of mitochondrial physiology and metabolism, telomere dysfunction, including shortening, was associated with impaired mitochondrial biogenesis and function, decreased gluconeogenesis, cardiomyopathy, and increased ROS [25].

Given the mounting evidence of possible combined associations of cancer risk with LTL and mtDNA, we 
sought to investigate, for the first time in the same study, the two markers with the addition of mtDNA deletion in order to gain a more comprehensive picture of their involvement in $\mathrm{BC}$ risk. Given the large number of caveats linked to these measurements as a first preliminary step, we wanted to determine how stable the measurements were. To do so, we isolated DNA from blood samples taken 15 years apart from 96 individuals. We observed a very good stability over time for both LTL (ICC 0.63) and mtDNA copy number (ICC 0.60). These values probably underestimate the real correlations because the starting material was not exactly the same: The DNA collected at baseline was isolated from buffy coat, whereas for the samples collected after 15 years, the extraction was done starting from whole blood.

In the cross-sectional analysis, our results suggest a clear association between longer telomeres and increased risk of $\mathrm{BC}$ independent of ER status. The association between longer telomeres and increased $\mathrm{BC}$ risk is biologically plausible because longer LTL may be a marker of an actively reproducing cell and therefore a cell that keeps dividing and that is exposed to an increased risk of acquiring tumor-causing mutations. The association between longer or shorter LTL and cancer risk could also be tumor-specific and may reflect the complex interaction between the genetic determination of telomere length and the environmental risk factors specific for each cancer type.

The analyses of our data did not show any strong association between mtDNA, when analyzed alone, and $\mathrm{BC}$ risk, contrary to what has been observed by others $[5,9$, 13, 18]. Authors of two recent meta-analyses investigated the role of mtDNA copy number in a large number of studies across different tumors and highlighted the great heterogeneity of the mtDNA effect in different cancer types and a null overall effect $[61,62]$. Interestingly, also the four studies in which researchers reported the association with $\mathrm{BC}$ showed a high degree of heterogeneity, possibly explaining the difference between those studies and our findings. However, when normalizing for mitochondrial deletions and using the mitochondrial integrity variable, we did observe an increase in risk with a higher number of functional copies. This association is biologically plausible because an increase in mtDNA copy number may reflect increased oxidative stress that in turn may increase damage levels, fuel inflammation [63], and finally increase the risk of developing BC. One of the novelties of our report is the introduction of a measure of mitochondrial genome integrity and the possibility of observing the effect of functional mitochondrial copy number in cancer risk instead of just measuring the effect mtDNA copy number regardless of their status. Given that when considering mtDNA copy number alone we did not see any association with $\mathrm{BC}$ risk, we hypothesize that this "correction," at least in part, could explain the differences and heterogeneity observed across previous reports. Analyzing the score of mtDNA and LTL, we observed an association with increased risk for the individuals with a high score; however, this probably reflects the association between LTL and $\mathrm{BC}$ risk.

This study has several clear strengths, one being its prospective design, which, to a certain extent, may reduce the possibility of reverse causation, which is a critical point when looking for the relationship between LTL, mtDNA, and cancer risk. A second strength is the possibility of avoiding possible confounders by adjusting for all known potential confounding factors on which information was available, such as smoking, BMI, and alcohol consumption. In addition, our data suggest that our calculations of the LTL and mtDNA ratios are unlikely to be heavily affected by sample handling or technical errors, given that even at 15 years apart the measurements remained comparable. A possible limitation of the present study is the relatively small sample size, which is partially counterbalanced, however, by the rather large number of $\mathrm{ER}-\mathrm{BC}$ cases.

\section{Conclusions}

We observed a very good correlation between the markers across a period of 15 years, highlighting how sample handling is crucial for this kind of analysis. Moreover, we also observed a strong and consistent association between longer telomere length and increased BC risk, and additionally, we found a novel association between the mitochondrial genome stability and $\mathrm{ER}+\mathrm{BC}$ risk.

\section{Additional files}

Additional file 1: Table S1. Correlation between the biomarkers in control subjects. (DOCX $13 \mathrm{~kb}$ )

Additional file 2: Table S2. Associations between $L T L$ and BC risk. (DOCX $15 \mathrm{~kb}$ )

Additional file 3: Table S3. Associations between mtDNA copy number and $B C$ risk. (DOCX $15 \mathrm{~kb}$ )

Additional file 4: Table S4. Associations between mtDNA integrity (ND4/ND1 divided by ND1/nuclear genome) and BC risk. (DOCX 15 kb)

\section{Abbreviations \\ BC: Breast cancer; BMI: Body mass index; CV: Coefficient of variation; EPIC: European Prospective Investigation into Cancer and Nutrition; ER: Estrogen receptor; ICC: Intraclass correlation; ICD-10: International Classification of Diseases, Tenth Revision; LTL: Leukocyte telomere length; mtDNA: Mitochondrial DNA; ND: Nicotinamide adenine dinucleotide dehydrogenase}

Acknowledgements

We thank the participants and the investigators of EPIC study.

\section{Funding}

The coordination of the EPIC study is financially supported by the European Commission (DG-SANCO) and the International Agency for Research on 
Cancer. The national cohorts are supported by the following organizations: Ligue Contre le Cancer, Institut Gustave Roussy, Mutuelle Générale de I'Education Nationale, Institut National de la Santé et de la Recherche Médicale (INSERM) (France); German Cancer Aid, German Cancer Research Center (DKFZ), Federal Ministry of Education and Research (BMBF) (Germany); the Hellenic Health Foundation, the Stavros Niarchos Foundation (Greece); Associazione Italiana per la Ricerca sul Cancro (AIRC) and National Research Council (Italy); Dutch Ministry of Public Health, Welfare and Sports (MWS), Netherlands Cancer Registry (NKR), LK Research Funds, Dutch Prevention Funds, Dutch ZON (Zorg Onderzoek Nederland), World Cancer Research Fund (WCRF), Statistics Netherlands (The Netherlands); Health Research Fund (FIS) (PI13/00061 to Granada, PI13/01162 to EPIC-Murcia), the regional Governments of Andalucía, Asturias, Basque Country, Murcia, and Navarra, and Instituto de Salud Carlos III (ISCIII) Redes temáticas de investigación cooperativa en salud (RETICS) (RD06/0020) (Spain); and Cancer Research UK (14136 to EPIC-Norfolk; C570/A16491 and C8221/A19170 to EPIC-Oxford) and the Medical Research Council (1000143 to EPIC-Norfolk, MR/M012190/1 to EPIC-Oxford) (United Kingdom).

\section{Availability of data and materials}

The datasets on which the present analyses are based are not publicly available, owing to incompatibility with the regulations of the different ethics committees in the contributing study centers. However, these datasets are available from the corresponding author upon reasonable request.

\section{Authors' contributions}

$D C$ performed the laboratory work. MB performed the statistical analyses. DC, $A S, M B, F C$, and RK drafted the manuscript. DC, FC, AS, and RK planned the study. DC, MB, AS, GS, LB, HO, RT, HBBdM, PHP, EW, MDC, MRB, AA, MG, LD, VK, GM, AT, RCT, FC, and RK collected the samples and the epidemiologic data. All authors read and approved the final manuscript.

\section{Ethics approval and consent to participate}

All individuals signed a written consent form, and the study was reviewed by ethics committees in the different study centers. The following are the ethics committees from the individual EPIC centers: University of Athens Medical School, Norwich District Ethics Committee, the National Committee on Health Research Ethics, Comité de Protection des Personnes, Ethics Committee of the Heidelberg University Medical School, Imperial College Research Ethics Committee, Comitato Etico Indipendente, Fondazione IRCCS Istituto Nazionale dei Tumori, Comitato Etico Locale Azienda Sanitaria di Firenze, Ethics Committee of Lund University, the Medical Ethics Committee of the University Medical Center Utrecht, regional ethics committee for northern Norway and the Norwegian Data Inspectorate, Scotland A Research Ethics Committee, Ethikkommission der Landesärztekammer Brandenburg Cottbus, Comité de Ética de Investigación Clínica (CEIC), Human Genetics Foundation Torino Ethics Committee, Umea Regional Ethical Review Board, and IARC Ethics Committee.

\section{Consent for publication}

Not applicable.

\section{Competing interests}

The authors declare that they have no competing interests.

\section{Publisher's Note}

Springer Nature remains neutral with regard to jurisdictional claims in published maps and institutional affiliations.

\section{Author details}

${ }^{1}$ Department of Biology, University of Pisa, Pisa, Italy. ${ }^{2}$ Division of Cancer Epidemiology, German Cancer Research Center/Deutsches Krebsforschungszentrum (DKFZ), Im Neuenheimer Feld 280, 69120 Heidelberg, Germany. ${ }^{3}$ Department of Experimental, Diagnostic and Specialty Medicine (DIMES), University of Bologna, Bologna, Italy. ${ }^{4}$ Centre de Recherche en épidémiologie et Santé des populations (CESP), Faculté de médecine Université Paris-Sud, Faculté de médecine - Université de Versailles Saint-Quentin-en-Yvelines (UVSQ), Institut national de la santé et de la recherche médicale (INSERM), Université Paris-Saclay, 94805 Villejuif, France. ${ }^{5}$ Institut Gustave Roussy, F-94805 Villejuif, France. ${ }^{6}$ Department of Clinical and Experimental Medicine, University of Pisa, Pisa, Italy. ${ }^{7}$ Cancer Registry and
Histopathology Department, "Civic - M.P. Arezzo" Hospital, Azienda Sanitaria Provinciale Di Ragusa, Ragusa, Italy. ${ }^{8}$ Department for Determinants of Chronic Diseases (DCD), National Institute for Public Health and the Environment (RIVM), PO Box 1, 3720 BA Bilthoven, The Netherlands. ${ }^{9}$ Department of Epidemiology and Biostatistics, The School of Public Health, Imperial College London, St. Mary's Campus, Norfolk Place, London W2 1PG, UK.

${ }^{10}$ Department of Social \& Preventive Medicine, Faculty of Medicine, University of Malaya, Pantai Valley, 50603 Kuala Lumpur, Malaysia.

${ }^{11}$ Department of Epidemiology, Julius Center for Health Sciences and Primary Care, University Medical Center Utrecht, Utrecht, The Netherlands.

${ }^{12}$ Department of Epidemiology and Biostatistics, Medical Research Council-Public Health England (MRC-PHE) Centre for Environment and Health, School of Public Health, Imperial College London, London, UK.

${ }^{13}$ Department of Community Medicine, Faculty of Health Sciences, University of Troms $\varnothing$, The Arctic University of Norway, Troms $\varnothing$, Norway. ${ }^{14}$ Department of Research, Cancer Registry of Norway, Institute of Population-Based Cancer Research, Oslo, Norway. ${ }^{15}$ Department of Medical Epidemiology and Biostatistics, Karolinska Institutet, Stockholm, Sweden. ${ }^{16}$ Genetic Epidemiology Group, Folkhälsan Research Center, Helsinki, Finland. ${ }^{17}$ Department of Epidemiology, Regional Health Council, Biomedical Research Institute of Murcia (IMIB-Arrixaca), Murcia, Spain. ${ }^{18}$ Consorcio de Investigación Biomédica en Red de Epidemiología y Salud Pública (CIBERESP), Madrid, Spain. ${ }^{19}$ Department of Health and Social Sciences, Universidad de Murcia, Murcia, Spain. ${ }^{20}$ Escuela Andaluza de Salud Pública, Instituto de Investigación Biosanitaria (ibs.GRANADA), Hospitales Universitarios de Granada/Universidad de Granada, Granada, Spain. ${ }^{21}$ Unit of Nutrition and Cancer, Bellvitge Biomedical Research Institute (IDIBELL), Catalan Institute of Oncology, L'Hospitalet de Llobregat, 08908 Barcelona, Spain. ${ }^{22}$ International Agency for Research on Cancer, Lyon, France.

${ }^{23}$ Epidemiology and Prevention Unit, Fondazione Istituto di Ricovero e Cura a Carattere Scientifico (IRCCS)-Istituto Nazionale dei Tumori, Via Venezian, 120133 Milan, Italy. ${ }^{24}$ Department Medical Sciences, University of Torino and Human Genetics Foundation (HuGeF), Torino, Italy. ${ }^{25}$ Hellenic Health Foundation, 11527 Athens, Greece. ${ }^{26}$ Cancer Epidemiology Unit, Nuffield Department of Population Health University of Oxford, Oxford OX3 ONR, UK.

${ }^{27}$ Genomic Epidemiology Group, German Cancer Research Center/Deutsches Krebsforschungszentrum (DKFZ), Heidelberg, Germany.

\section{Received: 10 August 2017 Accepted: 13 March 2018}

Published online: 17 April 2018

\section{References}

1. Wallace DC. A mitochondrial paradigm of metabolic and degenerative diseases, aging, and cancer: a dawn for evolutionary medicine. Annu Rev Genet. 2005;39:359-407.

2. Wallace DC. Bioenergetics and the epigenome: interface between the environment and genes in common diseases. Dev Disabil Res Rev. 2010; 16(2):114-9.

3. Clay Montier LL, Deng JJ, Bai Y. Number matters: control of mammalian mitochondrial DNA copy number. J Genet Genomics. 2009:36(3):125-31.

4. Veltri KL, Espiritu M, Singh G. Distinct genomic copy number in mitochondria of different mammalian organs. J Cell Physiol. 1990;143(1): 160-4.

5. Shen J, Platek M, Mahasneh A, Ambrosone CB, Zhao H. Mitochondrial copy number and risk of breast cancer: a pilot study. Mitochondrion. 2010;10(1): 62-8.

6. Bonner MR, Shen M, Liu CS, Divita M, He X, Lan Q. Mitochondrial DNA content and lung cancer risk in Xuan Wei, China. Lung Cancer. 2009;63(3): $331-4$.

7. Hosnijeh FS, Lan Q, Rothman N, San Liu C, Cheng WL, Nieters A, Guldberg P, Tjonneland A, Campa D, Martino A, et al. Mitochondrial DNA copy number and future risk of B-cell lymphoma in a nested case-control study in the prospective EPIC cohort. Blood. 2014;124(4):530-5.

8. Lan Q, Lim U, Liu CS, Weinstein SJ, Chanock S, Bonner MR, Virtamo J, Albanes D, Rothman N. A prospective study of mitochondrial DNA copy number and risk of non-Hodgkin lymphoma. Blood. 2008;1 12(10):4247-9.

9. Lemnrau A, Brook MN, Fletcher O, Coulson P, Tomczyk K, Jones M, Ashworth A, Swerdlow A, Orr N, Garcia-Closas M. Mitochondrial DNA copy number in peripheral blood cells and risk of developing breast cancer. Cancer Res. 2015;75(14):2844-50. 
10. Lynch SM, Weinstein SJ, Virtamo J, Lan Q, Liu CS, Cheng WL, Rothman N, Albanes D, Stolzenberg-Solomon RZ. Mitochondrial DNA copy number and pancreatic cancer in the Alpha-Tocopherol Beta Carotene Cancer Prevention. Cancer Prev Res (Phila). 2011;4(11):1912-9.

11. Qu F, Liu X, Zhou F, Yang H, Bao G, He X, Xing J. Association between mitochondrial DNA content in leukocytes and colorectal cancer risk: a casecontrol analysis. Cancer. 2011;117(14):3148-55.

12. Yu M. Generation, function and diagnostic value of mitochondrial DNA copy number alterations in human cancers. Life Sci. 2011;89(3-4):65-71.

13. Thyagarajan B, Wang $R$, Nelson $H$, Barcelo H, Koh WP, Yuan JM. Mitochondrial DNA copy number is associated with breast cancer risk. PLoS One. 2013;8(6):e65968.

14. Blein S, Berndt S, Joshi AD, Campa D, Ziegler RG, Riboli E, Cox DG. Factors associated with oxidative stress and cancer risk in the Breast and Prostate Cancer Cohort Consortium. Free Radic Res. 2014;48(3):380-6.

15. Cox DG, Dostal L, Hunter DJ, Le Marchand L, Hoover R, Ziegler RG, Thun MJ. $\mathrm{N}$-acetyltransferase 2 polymorphisms, tobacco smoking, and breast cancer risk in the breast and prostate cancer cohort consortium. Am J Epidemiol. 2011;174(11):1316-22.

16. Gonenc A, Erten D, Aslan S, Akinci M, Simsek B, Torun M. Lipid peroxidation and antioxidant status in blood and tissue of malignant breast tumor and benign breast disease. Cell Biol Int. 2006;30(4):376-80.

17. Sener DE, Gonenc A, Akinci M, Torun M. Lipid peroxidation and total antioxidant status in patients with breast cancer. Cell Biochem Funct. 2007; 25(4):377-82.

18. Shen J, Wan J, Song R, Zhao H. Peripheral blood mitochondrial DNA copy number, length heteroplasmy and breast cancer risk: a replication study. Carcinogenesis. 2015;36(11):1307-13.

19. Taylor RW, Turnbull DM. Mitochondrial DNA mutations in human disease. Nat Rev. 2005;6(5):389-402

20. Damas J, Samuels DC, Carneiro J, Amorim A, Pereira F. Mitochondrial DNA rearrangements in health and disease—a comprehensive study. Hum Mutat. 2014;35(1):1-14

21. Brierley EJ, Johnson MA, Lightowlers RN, James OF, Turnbull DM. Role of mitochondrial DNA mutations in human aging: implications for the central nervous system and muscle. Ann Neurol. 1998:43(2):217-23.

22. Chinnery PF, Samuels DC, Elson J, Turnbull DM. Accumulation of mitochondrial DNA mutations in ageing, cancer, and mitochondrial disease: is there a common mechanism? Lancet. 2002;360(9342):1323-5.

23. Nie H, Chen G, He J, Zhang F, Li M, Wang Q, Zhou H, Lyu J, Bai Y. Mitochondrial common deletion is elevated in blood of breast cancer patients mediated by oxidative stress. Mitochondrion. 2016:26:104-12.

24. Kim JH, Kim HK, Ko JH, Bang H, Lee DC. The relationship between leukocyte mitochondrial DNA copy number and telomere length in communitydwelling elderly women. PLoS One. 2013;8(6):e67227.

25. Sahin E, Colla S, Liesa M, Moslehi J, Muller FL, Guo M, Cooper M, Kotton D, Fabian AJ, Walkey C, et al. Telomere dysfunction induces metabolic and mitochondrial compromise. Nature. 2011;470(7334):359-65.

26. Hou L, Zhang X, Gawron AJ, Liu J. Surrogate tissue telomere length and cancer risk: shorter or longer? Cancer Lett. 2012;319(2):130-5.

27. Campa D, Martino A, Varkonyi J, Lesueur F, Jamroziak K, Landi S, Jurczyszyn A, Marques $H$, Andersen $V$, Jurado $M$, et al. Risk of multiple myeloma is associated with polymorphisms within telomerase genes and telomere length. Int J Cancer. 2015;136(5):E351-8.

28. Campa D, Mergarten B, De Vivo I, Boutron-Ruault MC, Racine A, Severi G, Nieters A, Katzke VA, Trichopoulou A, Yiannakouris N, et al. Leukocyte telomere length in relation to pancreatic cancer risk: a prospective study. Cancer Epidemiol Biomarkers Prev. 2014;23(11):2447-54.

29. Weischer M, Nordestgaard BG, Cawthon RM, Freiberg JJ, Tybjærg-Hansen A, Bojesen SE. Short telomere length, cancer survival, and cancer risk in 47102 individuals. J Natl Cancer Inst. 2013;105(7):459-68.

30. Qiu C, Enquobahrie DA, Gelaye B, Hevner K, Williams MA. The association between leukocyte telomere length and mitochondrial DNA copy number in pregnant women: a pilot study. Clin Lab. 2015;61(3-4):363-9.

31. Tyrka AR, Carpenter LL, Kao HT, Porton B, Philip NS, Ridout SJ, Ridout KK, Price LH. Association of telomere length and mitochondrial DNA copy number in a community sample of healthy adults. Exp Gerontol. 2015;66: 17-20.

32. Jung SJ, Cho JH, Park WJ, Heo YR, Lee JH. Telomere length is correlated with mitochondrial DNA copy number in intestinal, but not diffuse, gastric cancer. Oncol Lett. 2017;14(1):925-9.
33. Riboli E, Hunt KJ, Slimani N, Ferrari P, Norat T, Fahey M, Charrondiere UR, Hemon B, Casagrande C, Vignat J, et al. European Prospective Investigation into Cancer and Nutrition (EPIC): study populations and data collection. Public Health Nutr. 2002;5(6b):1113-24.

34. Kaaks R, Berrino F, Key T, Rinaldi S, Dossus L, Biessy C, Secreto G, Amiano P,

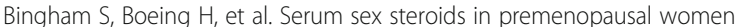
and breast cancer risk within the European Prospective Investigation into Cancer and Nutrition (EPIC). J Natl Cancer Inst. 2005;97(10):755-65.

35. Kaaks R, Johnson T, Tikk K, Sookthai D, Tjonneland A, Roswall N, Overvad K, Clavel-Chapelon F, Boutron-Ruault MC, Dossus L, et al. Insulin-like growth factor I and risk of breast cancer by age and hormone receptor status-a prospective study within the EPIC cohort. Int J Cancer. 2014;134(11):2683-90.

36. Kaaks R, Rinaldi S, Key TJ, Berrino F, Peeters PH, Biessy C, Dossus L, Lukanova A, Bingham S, Khaw KT, et al. Postmenopausal serum androgens, oestrogens and breast cancer risk: the European Prospective Investigation into Cancer and Nutrition. Endocr Relat Cancer. 2005;12(4):1071-82.

37. Kaaks R, Tikk K, Sookthai D, Schock H, Johnson T, Tiønneland A, Olsen A, Overvad K, Clavel-Chapelon F, Dossus L, et al. Premenopausal serum sex hormone levels in relation to breast cancer risk, overall and by hormone receptor status-results from the EPIC cohort. Int J Cancer. 2014;134(8): 1947-57.

38. Barrdahl M, Canzian F, Joshi AD, Travis RC, Chang-Claude J, Auer PL, Gapstur SM, Gaudet M, Diver WR, Henderson BE, et al. Post-GWAS geneenvironment interplay in breast cancer: results from the Breast and Prostate Cancer Cohort Consortium and a meta-analysis on 79,000 women. Hum Mol Genet. 2014;23(19):5260-70.

39. Barrdahl M, Canzian F, Lindstrom S, Shui I, Black A, Hoover RN, Ziegler RG, Buring JE, Chanock SJ, Diver WR, et al. Association of breast cancer risk loci with breast cancer survival. Int J Cancer. 2015;137(12):2837-45.

40. Campa D, Barrdahl M, Gaudet MM, Black A, Chanock SJ, Diver WR, Gapstur SM, Haiman C, Hankinson S, Hazra A, et al. Genetic risk variants associated with in situ breast cancer. Breast Cancer Res. 2015;17:82.

41. Campa D, Claus R, Dostal L, Stein A, Chang-Claude J, Meidtner K, Boeing H, Olsen A, Tjønneland A, Overvad K, et al. Variation in genes coding for AMPactivated protein kinase (AMPK) and breast cancer risk in the European Prospective Investigation on Cancer (EPIC). Breast Cancer Res Treat. 2011; 127(3):761-7.

42. Campa D, Husing A, McKay JD, Sinilnikova O, Vogel U, Tjonneland A, Overvad K, Stegger J, Clavel-Chapelon F, Chabbert-Buffet N, et al. The INSIG2 rs7566605 polymorphism is not associated with body mass index and breast cancer risk. BMC Cancer. 2010;10:563.

43. Campa D, McKay J, Sinilnikova O, Husing A, Vogel U, Hansen RD, Overvad K, Witt PM, Clavel-Chapelon F, Boutron-Ruault MC, et al. Genetic variation in genes of the fatty acid synthesis pathway and breast cancer risk. Breast Cancer Res Treat. 2009;118(3):565-74.

44. Garcia-Closas M, Couch FJ, Lindstrom S, Michailidou K, Schmidt MK, Brook MN, Orr N, Rhie SK, Riboli E, Feigelson HS, et al. Genome-wide association studies identify four ER negative-specific breast cancer risk loci. Nat Genet. 2013:45(4):392-8.e2.

45. Siddiq A, Couch FJ, Chen GK, Lindstrom S, Eccles D, Millikan RC, Michailidou K, Stram DO, Beckmann L, Rhie SK, et al. A meta-analysis of genome-wide association studies of breast cancer identifies two novel susceptibility loci at 6q14 and 20q11. Hum Mol Genet. 2012;21(24):5373-84.

46. Altilia S, Santoro A, Malagoli D, Lanzarini C, Ballesteros Alvarez JA, Galazzo G, Porter DC, Crocco P, Rose G, Passarino G, et al. TP53 codon 72 polymorphism affects accumulation of mtDNA damage in human cells. Aging. 2012;4(1):28-39.

47. He L, Chinnery PF, Durham SE, Blakely EL, Wardell TM, Borthwick GM, Taylor RW, Turnbull DM. Detection and quantification of mitochondrial DNA deletions in individual cells by real-time PCR. Nucleic Acids Res. 2002; 30(14):e68.

48. Rosner B. The intraclass correlation coefficient. In: Fundamentals of biostatistics. 6th ed. Belmont: Thomson-Brooks/Cole; 2006. p. 609-13.

49. Floegel A, Drogan D, Wang-Sattler R, Prehn C, Illig T, Adamski J, Joost HG, Boeing $H$, Pischon T. Reliability of serum metabolite concentrations over a 4-month period using a targeted metabolomic approach. PLoS One. 2012; 6(6):e21103

50. Brandon M, Baldi P. Wallace DC. Mitochondrial mutations in cancer. Oncogene. 2006;25(34):4647-62.

51. Yadav N, Chandra D. Mitochondrial DNA mutations and breast tumorigenesis. Biochim Biophys Acta. 2013;1836(2):336-44. 
52. Ebner S, Lang R, Mueller EE, Eder W, Oeller M, Moser A, Koller J, Paulweber B, Mayr JA, Sperl W, et al. Mitochondrial haplogroups, control region polymorphisms and malignant melanoma: a study in middle European Caucasians. PLoS One. 2011;6(12):e27192.

53. Lam ET, Bracci PM, Holly EA, Chu C, Poon A, Wan E, White K, Kwok PY, Pawlikowska L, Tranah GJ. Mitochondrial DNA sequence variation and risk of pancreatic cancer. Cancer Res. 2012;72(3):686-95.

54. Skonieczna K, Malyarchuk BA, Grzybowski T. The landscape of mitochondrial DNA variation in human colorectal cancer on the background of phylogenetic knowledge. Biochim Biophys Acta. 2012;1825(2):153-9.

55. McEachern MJ, Krauskopf A, Blackburn EH. Telomeres and their control. Annu Rev Genet. 2000;34:331-58.

56. de Lange T. Shelterin: the protein complex that shapes and safeguards human telomeres. Genes Dev. 2005;19:2100-10.

57. de Lange T. How telomeres solve the end-protection problem. Science. 2009;326:948-52

58. Martinez P, Blasco MA. Role of shelterin in cancer and aging. Aging Cell. 2010;9:653-66.

59. Bao D, Ba Y, Zhou F, Zhao J, Yang Q, Ge N, Guo X, Wu Z, Zhang H, Yang H, et al. Alterations of telomere length and mtDNA copy number are associated with overall survival in hepatocellular carcinoma patients treated with transarterial chemoembolization. Cancer Chemother Pharmacol. 2016; 78(4):791-9.

60. Alegria-Torres JA, Velazquez-Villafana M, Lopez-Gutierrez JM, ChagoyanMartinez MM, Rocha-Amador DO, Costilla-Salazar R, Garcia-Torres L. Association of leukocyte telomere length and mitochondrial DNA copy number in children from Salamanca, Mexico. Genet Test Mol Biomarkers. 2016;20(11):654-9.

61. Hu L, Yao X, Shen Y. Altered mitochondrial DNA copy number contributes to human cancer risk: evidence from an updated meta-analysis. Sci Rep. 2016;6:35859.

62. Mi J, Tian G, Liu S, Li X, Ni T, Zhang L, Wang B. The relationship between altered mitochondrial DNA copy number and cancer risk: a meta-analysis. Sci Rep. 2015;5:10039.

63. Kamp DW, Shacter E, Weitzman SA. Chronic inflammation and cancer: the role of the mitochondria. Oncology (Williston Park). 2011;25(5):400-10. 413

\section{Submit your next manuscript to BioMed Central and we will help you at every step:}

- We accept pre-submission inquiries

- Our selector tool helps you to find the most relevant journal

- We provide round the clock customer support

- Convenient online submission

- Thorough peer review

- Inclusion in PubMed and all major indexing services

- Maximum visibility for your research

Submit your manuscript at www.biomedcentral.com/submit 